\title{
Litiasis de Vesículas Seminales: un reto Diagnóstico y sus Avances Terapéuticos, A Propósito de un Caso
}

\section{Seminal Vesicle Stones: A Diagnosis Challenge and Their Therapeutic Advances, a Propos of a Case}

\author{
Leonel Felipe Mateus Acuña ${ }^{1}$ Javier Mauricio Salgado Tovar ${ }^{2}$ \\ ${ }^{1}$ Medico General Universidad del Rosario, Hospital Universitario \\ Address for correspondence Leonel Felipe Mateus Acuña, MD, \\ Mayor - Mederi, Bogotá, Colombia \\ 2 Urólogo Universidad Nacional de Colombia, Hospital \\ Universitario Mayor - Méderi, Bogotá, Colombia \\ Medico General Universidad del Rosario, Hospital Universitario \\ Mayor - Mederi, Av cll 63 \# 73-13, Bogotá., Colombia \\ (e-mail: felipematteusacu@gmail.com).
}

Urol Colomb 2018;27:187-190.

Resumen

Palabras Claves

- litiasis de vesícula seminal

- microambiente

- formación de cálculos

- obstrucción del tracto urinario

- dolor perineal

- ultrasonografía transrectal

- vesiculoscópia transuretral con láser
Introducción y Objetivos La litiasis en vesículas seminales es una entidad rara, diferentes alteraciones anatómicas que obstruyen los ductos eyaculadores favorecen la precipitación y formación de cálculos. Nuestro objetivo es actualizar el enfoque diagnóstico y terapéutico a propósito de un paciente con esa patología.

Materiales y Métodos Se describe el caso de un paciente de 42 años con sintomatología urinaria no específica, asociado a dolor perineal, diagnosticado posteriormente con Síndrome de Löfgren. Dada la persistencia de síntomas, se le realiza UROTAC con evidencia de calcificaciones bilaterales en vesículas seminales. Por tratarse de un hallazgo infrecuente y sintomático, realizamos una revisión de la literatura que nos permitiera orientar el tratamiento.

Resultados La litiasis vesicular se presenta con sintomatología poco específica. Existen ciertos factores que favorecen la formación de cálculos en las vesículas seminales como lo son el microambiente propio de las vesículas seminales y alteraciones anatómicas que favorecen la obstrucción del tracto urinario. El método de elección para el diagnóstico es la ultrasonografía transrectal, la cual se debe realizar en todos los pacientes con sospecha clínica y síntomas urinarios no específicos. El tratamiento de elección es la vesiculoscópia transuretral con litotricia. Actualmente se está evaluando la realización de la vesiculoscópia transuretral.

Conclusiones La litiasis de vesículas seminales es una entidad rara, con presentación clínica variable. Su diagnóstico y manejo se convierten en un desafío para el urólogo, debido a que no existe una alternativa de manejo estándar, el manejo dependerá de la experiencia en técnicas endourológicas y disponibilidad de equipos. received

December 1, 2016

accepted

July 7, 2017

published online

April 24, 2018
DOI https://doi.org/

$10.1055 / \mathrm{s}-0038-1645845$.

ISSN 0120-789X.

eISSN 2027-0119.
Copyright ( $)$ 2018, Sociedad Colombiana License terms de Urología. Publicado por Thieme Revinter Publicações Ltda., Rio de Janeiro, Brazil. Todos los derechos reservados.

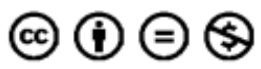




\begin{abstract}
Keywords

- seminal vesicle stones

- microenvironment

- stone formation

- urinary tract obstruction

- perineal pain

- trans rectal ultrasound

- transurethral seminal vesiculoscopy with laser

Purpose Seminal vesicle stones are a rare entity, different associations with anatomic variations like obstruction in ejaculatory ducts have relation with stone formation, these anatomic variations contribute to the aggregation of substances present in seminal liquid. Our purpose is to show a new diagnostic approach and treatment.

Materials and Methods The case of a 42-years-old man with a diagnosis of Lofgren syndrome, presented long standing non-specific urinary tract symptoms and perineal pain. Bilateral calcifications in seminal vesicle were observed using urotomography. With these uncommon findings and a symptomatic patient, a review of literature was performed for approach the treatment.

Results Seminal vesicle stone does not have specific symptomatology, and can be presented with perineal pain, infertility, painful ejaculation, irritative urinary symptoms. Different factors contribute to stone formation, the main factors are urinary tract obstruction, substances present in seminal liquid (fructose, prostaglandin), urinary reflux and recurrent infections. The gold standard for diagnosis is transrectal ultrasound.

Treatments have been changing during the years, the election treatment at the moment is transurethral seminal vesiculoscopy with laser, supported by different case reports in the literature.

Conclusions Seminal vesicle stones are rare entities, this pathology should be suspected with perineal pain, painful ejaculation and infertility. Many treatment techniques have been developed, however no standard procedure has been established to manage this entity yet, for this reason the diagnosis is a challenge to the urologist and the treatment depends on experience and equipment available.
\end{abstract}

\section{Introducción}

La litiasis en vesículas seminales es una entidad rara, son pocos los reportes de caso descritos en la literatura. Se ha relacionado con distintas infecciones del tracto urinario, alteraciones anatómicas que obstruyen los ductos eyaculadores favorece la agregación de sustancias propias del líquido seminal, precipitando la formación de cálculos. Su presentación clínica es variable y en muchos de los casos inespecífica. Nuestro objetivo es actualizar el enfoque diagnóstico y terapéutico a propósito de un paciente con esa patología.

\section{Caso Clínico}

Presentamos el caso de un paciente masculino de 42 años, quien en Julio del 2012, presentó cuadro de hematuria y disuria, diagnosticando uretritis, el cual se manejó con esquema antibiótico. Posteriormente cursó con dolor perineal tipo punzada de características fluctuantes, asociado a nicturia y tenesmo vesical, al examen físico y tacto rectal sin hallazgos relevantes. Se realizan paraclinicos iniciales, como único hallazgo relevante de los mismos, cultivo de semen positivo para E.coli multisensible, se indicó nuevo ciclo de esquema antibiótico y analgésico. Dada la persistencia de síntomas previamente descritos, se ampliaron estudios con ecografía renal y vías urinarias que arrojaron litiasis renal bilateral de $6 \mathrm{~mm}$ no obstructiva, a la cual se le dio manejo médico.

Meses después persistió con la sintomatología que cedía parcialmente con el manejo analgésico, adicionalmente cursó con dolor lumbar incapacitante por lo cual se realizó resonancia magnética de columna lumbosacra que reporta hernias discales L4, L5 y S1 sin compresión de raíces. Ocho semanas después, presentó cuadro de eritema nodoso con PCR elevado, diagnosticando de Síndrome de Löfgren.

El síndrome de Löfgren es una variante clínica de la sarcoidosis, con una prevalencia mayor en mujeres entre 30 y 40 años. Esa entidad es caracterizada por una afectación aguda de articulaciones, especialmente en miembros inferiores, además de la presencia de adenopatías hiliares bilaterales y eritema nodoso, sin embargo, un porcentaje de los pacientes solo se presenta con manifestaciones articulares o cutáneas. ${ }^{1,2}$

Su diagnóstico se realiza teniendo en cuenta tanto criterios clínicos, imagenológicos e histológicos. Existe un consenso actual para la realización del diagnóstico, el cual se puede realizar sin la necesidad de criterios histológicos. ${ }^{1}$ Es una entidad auto limitada, con resolución de síntomas de 6 a 12 meses en un $95 \%$ de los casos, ${ }^{2}$ el tratamiento está basado en el manejo sintomático con antiinflamatorios esteroideos, aunque en ciertos casos en que no hay respuesta al manejo sintomático, se prefiere el uso de corticoide para disminuir la respuesta inflamatoria aguda de esa variante clínica. ${ }^{1}$ 
Su evolución clínica no tuvo mejoría significativa a pesar del manejo analgésico, con aparición de nuevos síntomas como dolor con la eyaculación y pos eyaculatorio, razón por la cual se realizó UROTAC que reporta calcificaciones bilaterales en vesículas seminales, sin evidencia de litiasis renal y cistoscopia dentro de límites normales. Actualmente, el paciente persiste sintomático, sin aparición de nuevos síntomas, con periodos de intensificación de dolor perineal y síntomas descritos.

\section{Discusión}

La litiasis de vesículas seminales es una entidad rara, son pocos los reportes de caso publicados en la literatura, uno de los primeros reportes de caso publicados fue en el año 1928, posteriormente se han publicado cerca de 20 reportes de caso, de los cuales 4 de ellos están relacionados con cálculos gigantes en quistes de vesícula seminal. ${ }^{3}$ Dada su baja incidencia y la variedad de síntomas, hacen de esa entidad un reto diagnóstico.

No hay una certeza en cuanto a su epidemiología debido a los escasos reportes de caso y su dificultad diagnóstica. Se ha encontrado que la incidencia aumenta en pacientes mayores de 40 años y con comorbilidades tales como diabetes mellitus, prostatitis crónica, tuberculosis genitourinaria, esquistosomiasis, criptorquidia e hipospadias. ${ }^{3-5}$

Su etiología y fisiopatología son inciertas, se han postulado una serie de teorías, las cuales plantean que la formación de los cálculos es generada por la alteración en el microambiente propio de las vesículas seminales. Dentro de ellas se producen sustancias como fructosa, proteínas, prostaglandinas, así como sustancias mucoides cubiertas por sales como: fosfato de calcio, carbonato, urato, fosfato y estruvita. ${ }^{6}$ Schwartz propuso que, dentro de las vesículas seminales, pueden generarse calcificaciones de sales dentro de un núcleo de carbonato de apatita, ${ }^{4}$ de tal modo que existe susceptibilidad para la formación de cálculos por agregación de esas sustancias. Sumado a ese microambiente, se encuentran alteraciones anatómicas, infecciones, reflujo urinario u obstrucción del tracto urinario, lo cual favorece la alteración del microambiente de las vesículas seminales, aumentando así la susceptibilidad para la formación y agregación de dichas sustancias. En los casos reportados con el uso de la vesiculoscópia transuretral, se ha documentado que existen alteraciones como insuficiencia y disfuncionalidad del esfínter, al igual que obstrucción al flujo de salida de orina. ${ }^{7}$

Entre las enfermedades obstructivas de los conductos eyaculadores, se encuentran los cálculos en las vesículas seminales, que al igual que otras entidades clínicas, se caracterizan por el bajo volumen de eyaculación y por consiguiente infertilidad, principal evento clínico para el inicio de su estudio. Esos eventos obstructivos, pueden resultar de quistes provenientes de persistencia de conducto de Müller o de Wolf, atresia congénita de los conductos eyaculadores, calcificaciones en el Verum Montanum y cálculos en las vesículas seminales. ${ }^{8}$

El cuadro clínico es inespecífico, no hay evidencia de un síntoma cardinal para dicha patología, en los reportes de caso se encuentra que los pacientes pueden presentar síntomas como dolor perineal, dolor con la eyaculación, hematoespermia, hematuria, infecciones urinarias recurrentes, azoospermia, bajo volumen de eyaculación e infertilidad, resaltando que no siempre se presentan dichos síntomas. ${ }^{9}$ Un estudio realizado para evaluar la incidencia de cálculos en vesículas seminales en pacientes con hematoespermia, encontró que cerca del 17\% de los pacientes con hematoespermia, presentaban concomitantemente litos en esas estructuras. ${ }^{10}$ Otro estudio realizado en pacientes con hematoespermia concluyó que cerca del 95\% de los pacientes presentaban anormalidades anatómicas. ${ }^{11}$ Por lo tanto, tentativamente se podría establecer una relación entre alteraciones anatómicas, hematoespermia y formación de cálculos.

$\mathrm{Al}$ examen físico no hay un hallazgo relevante, el tacto rectal y tamaño prostático es normal en la mayoría de los casos reportados, pero puede encontrarse una masa de características pedregosas sin límites en algunos pacientes. ${ }^{8}$

La evaluación inicial se realiza por medio de estudios imagenológicos, en aquellos pacientes que presentan sintomatología inicial como hematoespermia, dolor perineal, dolor con la eyaculación e infertilidad..$^{9,12}$ Los estudios iniciales son la ecografía transrectal, tomografía abdominopélvica y resonancia magnética de pelvis. Esos estudios permiten evidenciar la presencia de cálculos, así como evaluar el grado de obstrucción y las opciones de tratamiento. Previamente, para la evaluación de los desórdenes de las vesículas seminales y próstata, se utilizaba la tomografía axial computarizada, sin embargo, actualmente se prefiere el uso de ultrasonografía transrectal para la evaluación de dichas estructuras, ya que brinda buenos resultados en la detección de cálculos, evitando así las preparaciones previas al examen o exposición a radiación. 9,11 Por esa razón, se considera la ultrasonografía transrectal como el Gold Estándar para el diagnóstico de litiasis de vesículas seminales y el primer paso en la evaluación de la obstrucción de los conductos eyaculadores. ${ }^{7,8,11}$ Cabe resaltar una de las ventajas que brinda la resonancia magnética frente a la ultrasonografía, la primera, revela la presencia de contenido hemorrágico en las vesículas seminales, siendo ese uno de los estudios de elección para evaluación en otras patologías de las glándulas sexuales masculinas accesorias. ${ }^{11}$

En cuanto al estudio paraclínico, el uso de uro dinamia y estudios funcionales como la cistouretrografía miccional y la uretrografía retrógrada, no son utilizados de forma rutinaria. Se ha visto que los pacientes son evaluados con diferentes paraclínicos como, análisis y cultivos de semen, antígeno prostático y uro cultivos, los cuales no tienen un soporte científico que recomiende su uso en la evaluación de los casos. ${ }^{7}$

El tratamiento ha evolucionado a través de los años, en los primeros reportes de caso, el tratamiento de elección era la vesicolectomía abierta. Esa intervención se relacionaba con altas tasas de complicaciones y morbilidad, debido a su complejo abordaje quirúrgico, por la localización anatómica de las vesículas seminales. La vesicolectomía está relacionada con un prolongado tiempo intraoperatorio, hemorragia y lesiones a órganos vecinos ${ }^{9}$; se han documentado 
complicaciones como epididimitis, eyaculación retrograda y lesión rectal. ${ }^{11}$ Por esa razón, fueron desarrolladas nuevas técnicas quirúrgicas entre las que se encuentran la vesiculostomía por laparoscopia con remoción de cálculos, vesiculectomía laparoscópica, así como la vesiculoscópia trans uretral, la cual fue inicialmente realizada en el año 1996. Posteriormente se realizó un reporte de caso de litotricia endoscópica en el año 2005 in vivo, con resultados exitosos. ${ }^{7}$ En un estudio realizado a 12 pacientes con un promedio de edad entre 25 y 57 años, quienes presentaban cuadro de hematoespermia, se les realizó manejo quirúrgico con vesiculoscópia trans uretral retrograda, utilizando un ureteroscópio rígido de $7.3 \mathrm{Fr}$, bajo visión directa, en el cual se hallaron cálculos de 1 a $5 \mathrm{~mm}$ de diámetro en las vesículas seminales, los cuales fueron removidos con alta tasa de éxito, presentándose complicaciones como sangrado, epididimitis, prostatitis, eyaculación retrograda e incontinencia urinaria, todas ellas en baja frecuencia. ${ }^{11}$

Por eso, el tratamiento de elección para la litiasis de vesículas seminales basado en los diferentes reportes de caso, nuevas técnicas y abordajes quirúrgicos, es la vesiculoscópia transuretral con litotricia. Ese abordaje quirúrgico se ha implementado en los últimos reportes de caso publicados, demostrando una buena tasa de resolución de síntomas, baja instancia hospitalaria, menos tiempo y complicaciones intra operatorias, ni postoperatorias, ${ }^{6,7,9,11}$ por lo tanto, ese nuevo abordaje quirúrgico mínimamente invasivo es seguro y efectivo para la resolución de la litiasis de vesículas seminales. Cabe resaltar que el abordaje endoscópico transuretral está descrito en aquellos pacientes que presentan calcificaciones de pequeño diámetro (menor de $3.5 \mathrm{~cm}$ ), y no presentan dilatación o quistes en las vesículas seminales, en los pacientes que presentan quistes o dilatación, el abordaje de elección es el laparoscópico transperitoneal, debido a que se logra la extracción exitosa de los cálculos y corrección de las vesicular seminales dilatadas. ${ }^{12}$

\section{Conclusión}

La litiasis de vesículas seminales es una entidad rara, donde su etiología y fisiopatología no está dilucidada, siendo un reto diagnóstico. Se han relacionado diferentes alteraciones anatómicas que favorecen la formación y agregación de las sustancias propias de las vesículas seminales por la alteración en el microambiente. El Gold estándar para su diagnóstico es la ultrasonografía transrectal, debido a que no necesita preparación previa al examen ni exposición a la radiación. El tratamiento ha evolucionado, siendo actualmente el más utilizado la vesiculoscópia transuretral con litotricia, por su buena tasa de resolución de síntomas, bajas tasas de complicación y morbilidad.

\section{Responsabilidades Éticas}

Protección de personas y animales.

Los autores declaran que para esta investigación, no se han realizado experimentos en seres humanos ni en animales.

Confidencialidad de los datos.

Los autores declaran que han seguido los protocolos de su centro de trabajo sobre la publicación de datos de pacientes.

Derecho a la privacidad y consentimiento informado.

Los autores declaran que en este artículo no aparecen datos de pacientes.

Conflicto de Intereses

Los autores declaran no tener ningún conflicto de intereses.

\section{Bibliografía}

1 Tejera Segura B, Holgado S, Mateo L, Pego-Reigosa JM, Carnicero Iglesias M, Olivé A. Síndrome de Löfgren: estudio de 80 casos. Med Clin (Barc) 2014;143(04):166-169

2 Villanueva M, Kurnat Y, Vázquez J, Gracia MP, Galindo MC. Síndrome de Löfgren. A propósito de un caso. Semergen 2009; 35(05):249-251

3 Singh I, Ansari MS. Idiopathic bilateral giant seminal vesicle calculi and calcification of the male ejaculatory system: Current review of diagnosis and management. Indian J Surg 2006;68(01): 38-40 [Case Report]

4 Hadidi M, Hadidy A, Alrabadi AF, Ahdul-Wahab AD, Murshidi MM. Bilateral very large calcium oxalate stones in the seminal vesicles: case report and literature review. Urol Res 2011;39(06):509-513 10.1007/s00240-011-0371-0

5 Herts BR. Calcification of the Seminal Vesicles. J Urol 2015;194 (01):209-211

6 Ozgok Y, Kilciler M, Aydur E, et al. Endoscopical seminal vesicle stone removal. Urology 2005;(65):591.e9-591.e12 10.1016/j. urology.2004.09.015

7 Lee TH, Juan Y-S, Jang M-Y, Wang H-S, Shen J-T. Successful seminal vesiculoscopic lithotripsy of seminal vesicle stone: A case report and literature review. Urol Sci 2014;25(04):134-136

8 Smith JF, Walsh TJ, Turek PJ. Ejaculatory duct obstruction. Urol Clin North Am 2008;35(02):221-227, viii 10.1016/j.ucl.2008.01.011

9 Han P, Yang YR, Zhang XY, Wei Q. Laparoscopic treatment of a calcium fluorophosphate stone within a seminal vesicle cyst. Asian J Androl 2008;10(02):337-340

10 Juan U, Juan G. Actualización en hematoespermia. Urol Colomb 2009;18:49-62

11 Song T, Zhang X, Zhang L, Zhang F, Fu WJ. Transurethral seminal vesiculoscopy in the diagnosis and treatment of seminal vesicle stones. Chin Med J (Engl) 2012;125(08):1475-1478 10.3760/cma. j.issn.0366-6999.2012.08.021

12 Yun S, Kim T, Kwon W, et al. A large Stone in dilated left seminal vesicle: laparoscopic removal and partial seminal vesiculectomy. Korean J Urol 2008;49:656-658 